\title{
Preface
}

\section{Mathematical Virology}

This issue of the Journal of Theoretical Medicine is devoted to the proceedings of the Workshop on Mathematical Virology, organised jointly by Philip Maini, Tom McLeish, Peter Stockley and Reidun Twarock at the Mathematical Institute in Oxford in June 2004. It was a satellite workshop of the Isaac Newton Institute Programme "Statistical Mechanics of Molecular and Cellular Biological Systems" that focussed on the mathematics, biology and biophysics of vital stages of the viral life cycle that are key for advances in the design of anti-viral therapeutics.

The workshop created an interdisciplinary environment in which the synergies of existing approaches could be explored and novel avenues of research be identified. During the workshop, one of the senior biological contributors ruefully confided that he had attended many interdisciplinary workshops where physical and biological scientists are invited to communicate their approaches to problems, but that this was the first in his experience that had "worked". By "working" he could not have meant that all participants saw uniformly eye-to-eye-we had our share of the classic paradigm conflicts of preferring general phenomena on the one hand and specific molecular structures on the other! Not everyone agreed on which questions were interesting or useful. However, the controlled size of the workshop complemented a remarkable readiness to listen on behalf of the participants. This allowed the discussions to be enriched by many years of reflection on our topic on the part of some, and by the freshness of approach of newcomers to the field. The real test will come in the results, of which this volume marks just the first fruits.

The contributions are collected in this volume as a stateof-the-art account of the field. The division into three main parts reflects three major challenges: The understanding of the structure of viral capsids, i.e. of the protein shells that encapsulate and hence provide protection for the viral genome, their assembly, and the packaging of the genetic material. The summary below is a brief guide for the reader, highlighting the main achievements and indicating connections between different approaches. For most speakers who were not able to submit a contribution, an extended abstract is available at the end of this volume to prompt the interested reader to the corresponding publications.
The first part of the volume is devoted to the structural aspects of viral capsids. David Stuart, a local guest speaker, introduced the topic and illustrated the current state-of-the-art with his group's crystallographic data. Despite the ground breaking work of Caspar and Klug in the 1960s that laid the foundation for the analysis and classification of viral capsids, many important questions remain open. One of them concerns the origin of icosahedral symmetry in viruses. Robijn Bruinsma addresses this question, and provides a theory for the explanation of the fact that over $50 \%$ of the known viruses exhibit icosahedrally symmetric capsids.

Moreover, growing experimental evidence has shown that hexagonal lattices and triangulations, which are at the heart of the principle of quasi-equivalence in Caspar-Klug Theory, are not sufficient to describe the surface structures of all viral capsids. Suggestions for alternative models are presented by Russell Schwartz, who reports on the local rules approach for encoding information on the local environments of the capsid proteins, and by Reidun Twarock, who discusses mathematical models for the locations of the protein subunits and inter-subunits bonds in viral capsids based on tiling theory.

As we learned from Harri Jäälinoja in his report on a cryo-electron microscopy study of an archaeal Haloarcula virus (SH1), the variety of viruses with unexplained surface structures is far from being exhausted, and many exciting discoveries are still to be expected along these lines. Novel mathematical techniques for their description are needed, such as for example a model that accounts for the arrangement of the dimers and trimers in this $T=28$ virus. Similarly, structural peculiarities of viral capsids, such as the chainmail structure of head II of bacteriophage HK97 reported by Roger Hendrix, call for an extended mathematical framework that incorporates them. A description of the chainmail structure in bacteriophage HK97 via tiling theory is suggested in the contribution of Reidun Twarock.

The elasticity properties of viral capsids were considered by Charlie Brooks, who used an elastic network normal mode analysis to explore mechanical, physical and chemical aspects of virus swelling, by David Nelson, who considered buckling transitions in spherical shells based on the nonlinear physics of thin elastic shells, and by Fred MacKintosh, who discussed the elastic 
response of microtubules and bacteriophage capsids in view of the measurement of local material properties of these structures. The stability of viral capsids was addressed by Mike Thorpe from the point of view of constraint theory, exploring the rigidity of the capsids.

Another key area in virology is viral capsid assembly. Of particular interest are the building blocks of assembly, the structural properties of intermediates as well as the pathways along which assembly takes place. Different guiding principles for a description of the kinetics of viral self-assembly have been explored. David Wales reports on viral self-assembly guided by a potential energy surface. An interpretation of virus assembly reactions in the framework of minimal thermodynamic-kinetic models is provided by Adam Ziotnick. His model is restricted to certain types of viruses, and a generalisation to Papovaviridae is discussed by Tom Keef based on the tiling approach outlined by Reidun Twarock in the section on virus structure. Moreover, the structural equivalence of molecular forms of Carbon called fullerenes and viral capsids lends itself to a transfer and exploitation of the corresponding mathematical techniques. Such a combinatorial approach is presented by Richard Kerner in his stochastic models of icosahedral capsid growth. Moreover, the contribution of Robijn Bruinsma mentioned in the section on virus structure provides a further equilibrium approach to viral self-assembly.

A close-up look at the mechanisms controlling in vitro assembly of bacteriophages was provided by Roman Tuma. Based on the example of bacteriophage P22 he showed that the assembly kinetics, which are crucial for the outcome of the assembly process, can be modulated via dimerization of the scaffolding protein. Since assembly depends on the association constants between the capsid proteins, knowledge of the structure of the individual proteins is crucial for an exploitation of the assembly models in the framework of anti-viral drug design. The molecular dynamics studies of Paolo Carloni, that provide a structural analysis of capsid proteins, cover this important aspect.

Of particular interest during viral capsid assembly are lattice transformations that occur in the surface structures of the capsids during maturation. Jonathan King discusses lattice transformations during scaffold-mediated assembly, i.e. for assembly guided by scaffold proteins based on the examples of bacteriophage P22 and HK97. The dramatic rearrangements of bacteriophage HK97 head assembly have furthermore been described by Roger Hendrix. Note that the results of David Nelson mentioned above are important for modelling the maturation.

A further vital stage of the viral life cycle is the packaging of the viral genome. The statics and dynamics of DNA and polymer packaging are considered by Davide Marenduzzo, and a movie illustrating their results can be found on the webpage of the Journal of Theoretical Medicine. A close-up look at the packaging motor of bacteriophage $\phi 29$ is provided by Nicola Stonehouse. Genome packaging is discussed by Peter Stockley in connection with the assembly of RNA bacteriophages.

We would like to thank everyone who helped to make this event possible, especially the Mathematical Institute in Oxford for their hospitality and Charlotte Rimmer for her help with the local organisation. Generous financial support by the EPSRC, the London Mathematical Society and the Isaac Newton Institute for the Mathematical Sciences in Cambridge is gratefully acknowledged. We also would like to thank Roger Hendrix for providing the conference photo, which can be found at the end of this volume.

Tom McLeish, Peter Stockley and Reidun Twarock

March 2005 


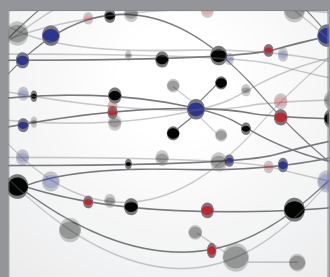

The Scientific World Journal
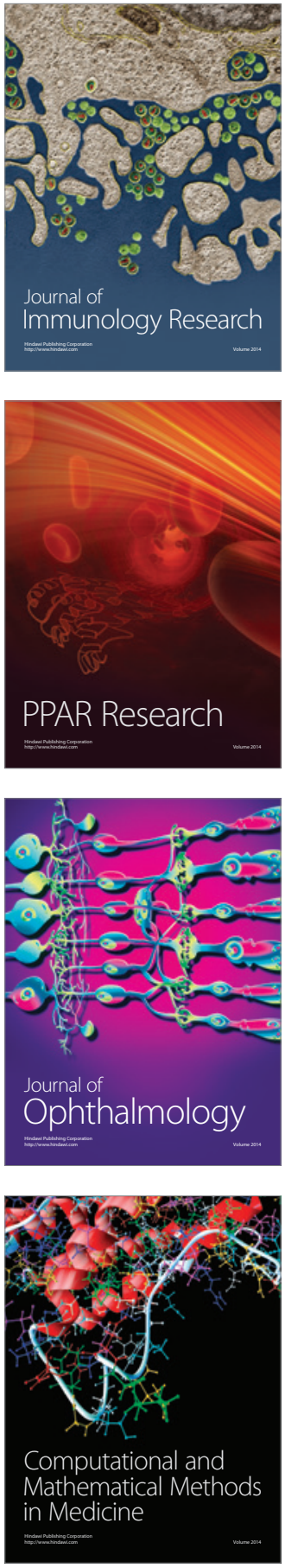

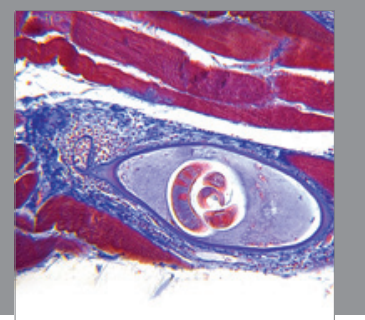

Gastroenterology

Research and Practice
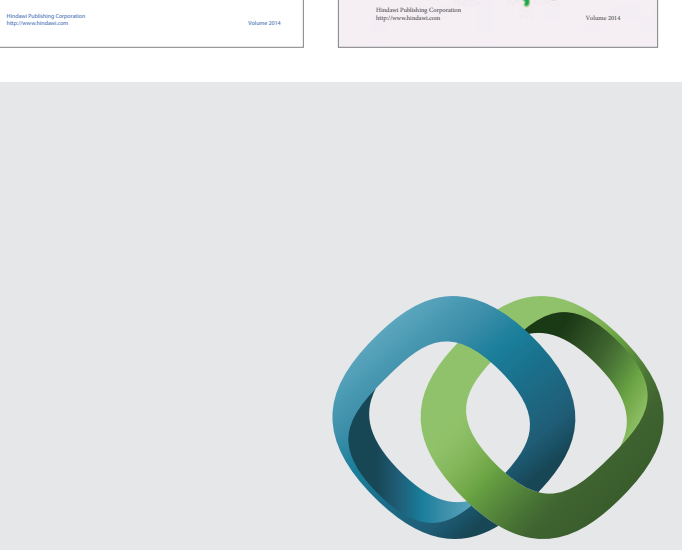

\section{Hindawi}

Submit your manuscripts at

http://www.hindawi.com
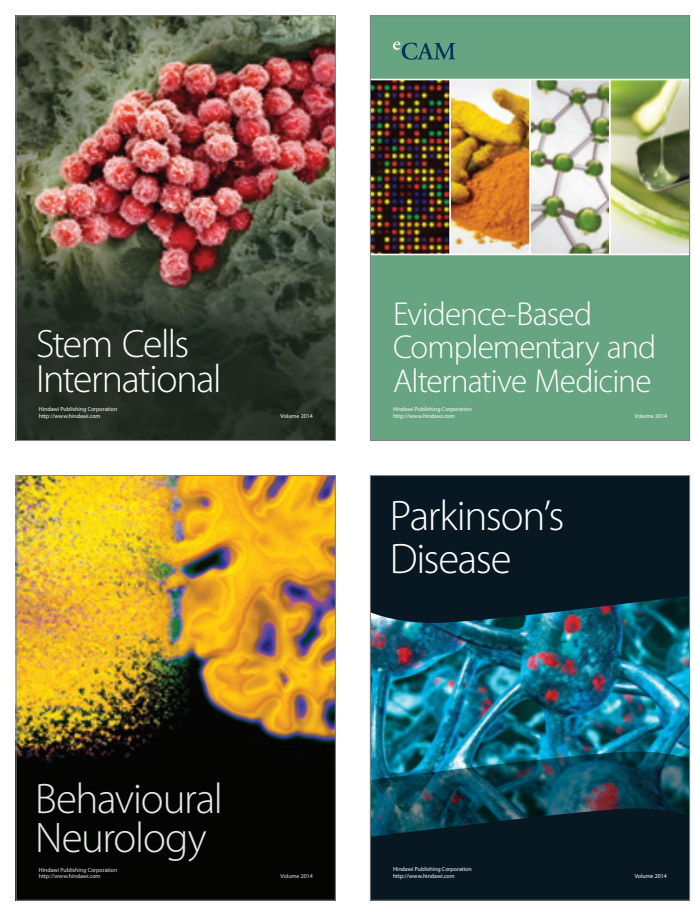

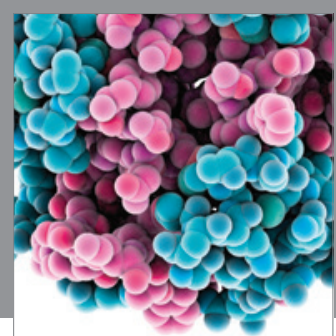

Journal of
Diabetes Research

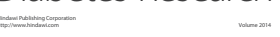

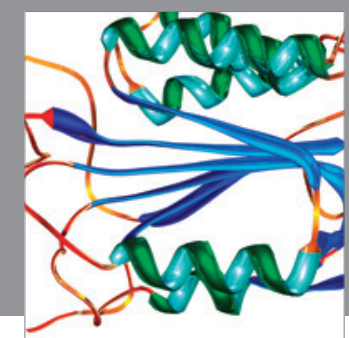

Disease Markers
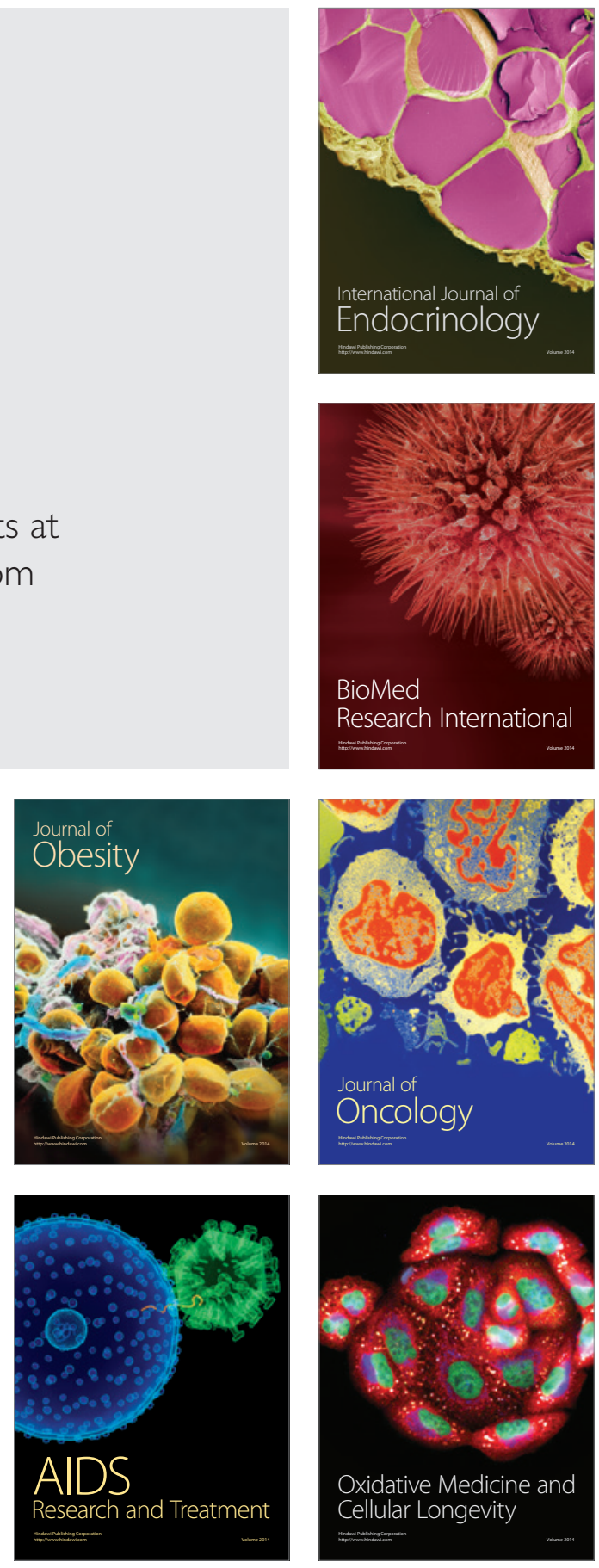